\title{
Monitoring of physiological parameters during high frequency ventilation $(\mathrm{HFV})$
}

\author{
Björn Jonson, Burkhard Lachmann' and Roger Fletcher \\ Departments of Clinical Physiology and Anaesthesia, University of Lund, Lund, Sweden, and 'Department of Anaesthesia, Erasmus \\ University, Rotterdam, The Netherlands
}

\begin{abstract}
Non-biological descriptors such as jet feeding pressure and oscillator stroke volume are often used to describe HFV. This results in confusion and hinders acceptance of HFV. The goal of this paper is to show how physiological parameters which are valid during $\mathrm{HFV}$ can be monitored. Airway pressure measured in narrow tubes with high linear flow rates is underestimated. A relevant airway pressure must be measured well below the tracheal tube. Pressure measured higher up should be validated against peripheral pressure measurements. Minute ventilation and expired $\mathrm{CO}_{2}$ concentration can be determined with a ServoVentilator and a $\mathrm{CO}_{2}$ analyzer arranged at its exit port. Minute ventilation and $\mathrm{CO}_{2}$ elimination can thereby be continuously monitored during high frequency jet ventilation or so-called "combined high frequency jet ventilation" to prevent undetected disturbance of ventilation and perfusion. Physiological dead space can be studied for optimization of ventilatory pattern. The principle of gas analysis at the exit port of the ventilator may be used for FRC determinations with sulfur-hexafluoride.
\end{abstract}

Key zvards: Airway pressure; dead space; functional residual capacity; gas exchange; high frequency ventilation; minute ventilation; monitoring.

When various modes of HFV are described, non-physiological terms have often been used rather than traditional parameters such as tidal volume, minute ventilation, alveolar ventilation and physiological dead space. As emphasized by Dr Wagner (in this volume) most traditional parameters are also valid during HFV. Undue discarding of traditional measures hampers the understanding and thereby clinical use of HFV. Misconceptions and difficulties in monitoring physiological parameters during HFV may explain why purely technical data, such as the feeding pressure of the jet, have replaced biologically significant data.

As monitoring during HFV has been poorly developed, ventilation has often been based upon "rule of thumb", personal clinical experience and intermittent sampling of arterial blood. Even under circumstances where HFV offers documented advantages, its general acceptance seems unlikely until means of monitoring ventilation, and particularly gas exchange have been devised. HFV is not an easy technique to apply, and it is often used in clinically complicated situations. It appears that continuous monitoring of gas exchange, e.g. the rate of $\mathrm{CO}_{2}$ elimination $\left(\mathrm{VCO}_{2}\right)$, is of particular value, as alveolar ventilation and perfusion are guaranteed as long as $\mathrm{VCO}_{2}$ is adequate.

The goal of the present paper is to demonstrate that monitoring of minute ventilation and $\mathrm{CO}_{2}$ elimination can be performed with standard equipment. The efficiency of various modes of $\mathrm{HFV}$ can easily be ap- praised. When airway pressure is monitored, the Bernoulli effect must be recognized. Finally, some hints are presented as to how functional residual capacity (FRC) may be measured during HFV.

\section{EQUIPMENT}

High frequency jet ventilation (HFJV), or combined high frequency jet ventilation (CHFJV) was administered with a Servo Ventilator 900 or $900 \mathrm{C}$ combined with a prototype Servo High Frequency Unit 970 (Siemens-Elema, Sweden). The latter unit produces jet pulses during a desired phase of the Servo Ventilator. The jet ventilation was delivered via the jet orifice of the Mallinckrodt Hi-Lo Jet tube (no. 8 or 9). The main lumen of the tube was connected to the Servo Ventilator.

In dog and pig studies of various modes of jet ventilation in health and in the respiratory distress syndrome, an anaesthetic bag was included in the expiratory line (Fig. 1). The bag serves as a reservoir and mixing chamber for expiratory gas. It allows free entrainment of gas during HFJV. During ordinary HFJV the Servo Ventilator was used as a system for measurement of minute ventilation. It was set at a frequency of $60 \mathrm{~min}^{-i}$ and an inspiratory time of $20 \%$. Pause time was set at zero. During the short inspirations, the pressure in the anaesthetic bag did not increase significantly. At the exit port of the ventilator the cuvette of the $\mathrm{CO}_{2}$ Analyzer 930 (Siemens-Elema, Solna, Sweden) was connected. This analyzer was used to measure $\mathrm{CO}_{2}$ concentration in mixed expired gas, which is continuously displayed as "end tidal $\mathrm{CO}_{2}$ concentration". It also continuously displays the rate of $\mathrm{CO}_{2}$ elimination in $\mathrm{ml} / \mathrm{min}$. This information is obtained by integration of the product (flow $\times \mathrm{CO}_{2}$ concentration), automatically performed in the analyzer.

The $\mathrm{CO}_{2}$ cuvette is flushed with fresh inspiratory gas during inspiration. This allows a correct resetting of the $\mathrm{CO}_{2}$ analyzer, which automatically performs during each inspiratory cycle of the venti- 


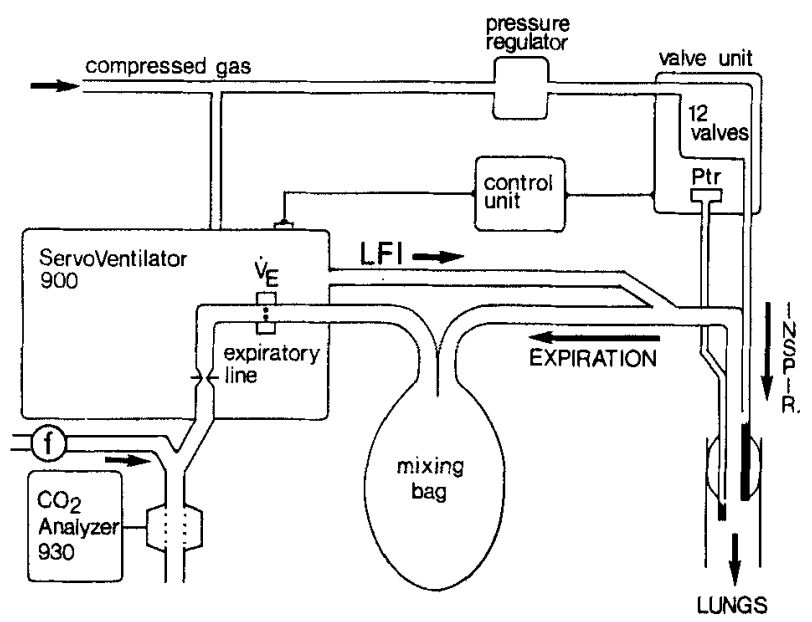

Fig. 1. When HFJV is the only or main component of ventilation, the Servo Ventilator serves mostly as a measuring and monitoring device. Alarm limits can be set for minute ventilation. A large anaesthetic bag in the expiratory limb may be used as storage for expired gas during short inspiratory phases of the Servo Ventilator. It will also permit free entrainment of gas during the jet pulses. The cuvette of the $\mathrm{CO}_{2}$ Analyzer 930 may be attached to the expiratory port of the Servo Ventilator. The cuvette is flushed during the inspiratory phase with fresh gas to allow the automatic zero reset function. The valve $(f)$ is a modified calibrating device for the analyzer. If desired, the Servo Ventilator may be set to deliver a low frequency component of ventilation ( $\mathrm{LFI}=$ low frequency inflations), that will be superimposed on HFJV.

lator. If the inspiratory line of the Servo Ventilator is not used for CHFJV, it may be used to flush the cuvette. At CHFJV we have instead used the device for calibration of the analyzer with its solenoid valve. Its socket connection was modified so that the valve opened during inspiration and not during expiration. The valve was fed with gas from the mixer of the ventilator via a pressure regulating valve.

In preliminary laboratory attempts to determine FRC during HFJV a cuvette for analysis of sulfur-hexafluoride $\left(\mathrm{SF}_{6}\right)$ was attached at the ventilator exit port in the same way as the $\mathrm{CO}_{2}$ Analyzer. $\mathrm{SF}_{6}$ has previously been shown to be suitable for gas washout studies during artificial ventilation $(2,3)$.

During CHFJV the $\mathrm{CO}_{2}$ Analyzer may be used in the standard mode (Fig. 2). In a study of expiratory flushing of large airways (EFLA) the $\mathrm{CO}_{2}$ signal and the flow signal from the Servo Ventilator were fed to a computer system. The whole system was integrated into a "Servo Ventilator 900I" which is a scientific set-up (SiemensElema). The signals were processed to yield the single breath test for $\mathrm{CO}_{2}$, SBT $-\mathrm{CO}_{2},(4)$.

The High Frequency ventilator contains a pressure transducer allowing pressure to be measured via the pressure lumen of the tracheal tube. This lumen ends within the tube close to its tip. The pressure measured in the tube was compared to pressure measured in the trachea and airways down to segmental bronchi. The pressure was measured with a Millar transducer tip catheter, F5. Pressure was also measured with the retrograde catheter technique (5), which yields the pressure to which airways of $2-3 \mathrm{~mm}$ diameter are exposed. All pressure transducers were carefully checked with regard to frequency response, which was flat to $60 \mathrm{~Hz}$ or more.

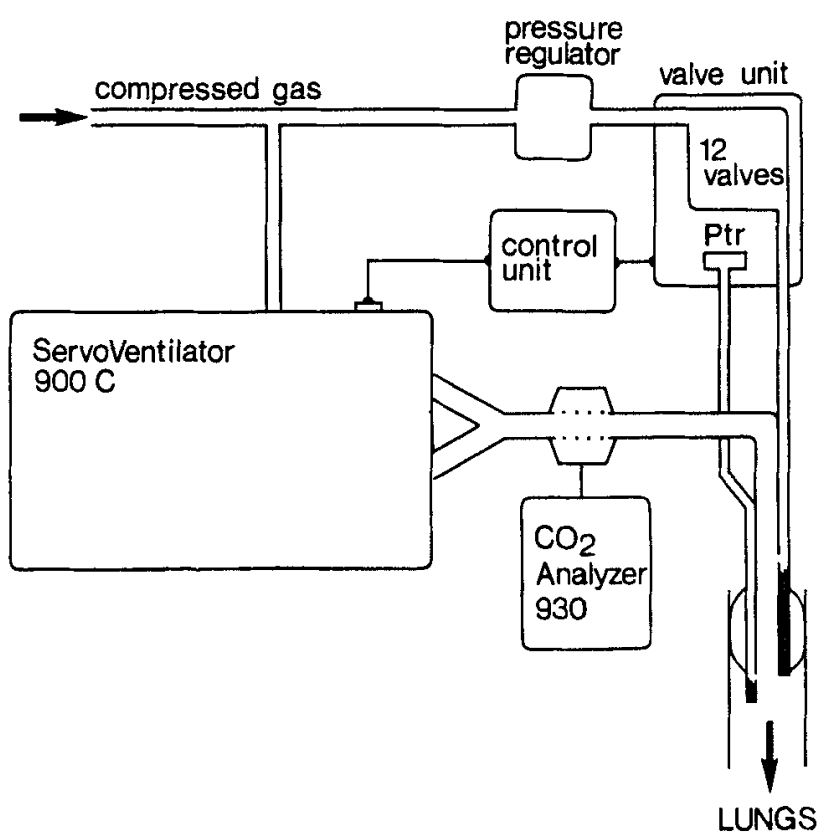

Fig. 2. During some modes of CHFJV such as EFLA the $\mathrm{CO}_{2}$ analyzer 930 is used in a conventional mode. This set-up allows recording of the single breath test for $\mathrm{CO}_{2}$ (Fig. 4).

\section{RESULTS}

The results from various studies are reviewed in order to illustrate the feasibility of physiological monitoring during HFJV.

\section{Physiological dead space during HFJV}

Healthy pigs were studied in order to evaluate how physiological dead space fraction ( $V_{D}$ phys) depends upon frequency and I:E ratio in HFJV (6). The equipment set-up is shown in Fig. 1.

When the mode of HFJV was changed, ventilation was immediately adjusted so that the rate of $\mathrm{CO}_{2}$ elimination $\left(\dot{\mathrm{VCO}}_{2}\right)$ was maintained at its steady state level observed during ordinary mechanical ventilation. Extensive experiments with widely differing HFJV settings demanded threefold variations in minute ventilation. The continuous monitoring of $\mathrm{CO}_{2}$ elimination made maintenance of isocapnia easy when the ventilator was reset. Adjustment of ventilation due to changes in arterial partial pressure of $\mathrm{CO}_{2}\left(\mathrm{PaCO}_{2}\right)$ were seldom required.

From $\mathrm{PaCO}_{2}$ and mixed expired $\mathrm{CO}_{2}\left(\mathrm{P}_{\mathrm{E}} \mathrm{CO}_{2}\right) \mathrm{V}_{\mathrm{D}}$ phys was calculated:

$$
\mathrm{V}_{\mathrm{Dphys}}=1-\mathrm{P}_{\mathrm{E} \mathrm{CO}_{2}} / \mathrm{PaCO}_{2}
$$

Table 1 shows some results. The lowest dead space fraction was found at the lowest HFJV frequency, i.e. $2 \mathrm{~Hz}$, and when the $\mathrm{I}: \mathrm{E}$ ratio was lowest, i.e. $1: 20$. 
Table 2

Physiological deadspace (\%) at different modes of HFJV.

\begin{tabular}{rcccc}
\hline & \multicolumn{4}{c}{ Frequency $(\mathrm{Hz})$} \\
\cline { 2 - 5 } Ti & 2 & 4 & 7 & 11 \\
\hline 5 & 32 & 45 & 58 & 73 \\
10 & 38 & 49 & 61 & 74 \\
15 & 43 & 52 & 64 & 74 \\
20 & 48 & 56 & 68 & 79
\end{tabular}

Ti is the duration of jet pulses in $\%$ of the cycle $(n=12)$.

For monitoring purposes the accuracy of the value of $\mathrm{CO}_{2}$ elimination presented continuously to the analyzer has been found to be adequate. It is, however, slightly lower than the true value. The reason is that a small volume of mixed expired gas is left in the $\mathrm{Y}$ piece between the ventilator and the $\mathrm{CO}_{2}$ cuvette. The $\mathrm{CO}_{2}$ content of this gas is flushed out by the fresh gas flow during the inspiratory phase and is not included in the monitored value. It should be noted that the fresh gas flow must be affluent in order to eliminate $\mathrm{CO}_{2}$ completely from the cuvette. For scientific measurement of $\mathrm{CO}_{2}$ elimination, this parameter is calculated from expired minute ventilation read on the digital display of the Servo Ventilator and the fraction of $\mathrm{CO}_{2}$ in expired gas read as "end-tidal $\mathrm{CO}_{2}$ " on the $\mathrm{CO}_{2}$ analyzer.

\section{Expiratory flushing of airway dead space (EFLA) in CHFJV}

Healthy dogs were studied with the set-up shown in Fig. 2 (7). Various modes of CHFJV were studied. Jet pulses of fresh gas were delivered during the late part of expiration in order to flush out dead space gas from the large airways. Isocapnia was maintained at changes of ventilatory pattern according to the same principle as described above - and as easily. When EFLA was given as four pulses during the last second

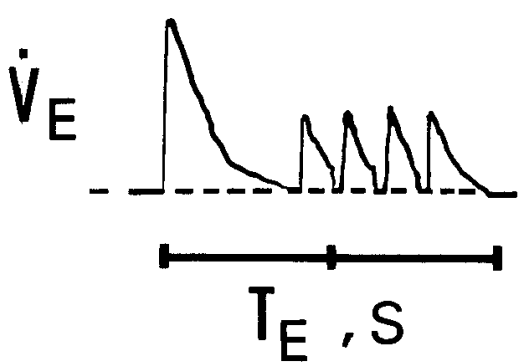

Fig. 3. During EFLA jet pulses are delivered during the late part of expiration via the HFU (Fig. 2). The expiratory flow recording $\left(\dot{V}_{E}\right)$ against expiratory time $\left(T_{E}\right)$ recorded with the ventilator flow meter shows here four "mini-expirations" corresponding to four pulses. Note that some time is allowed for expiration of the last pulse before the next inspiration.
Effects of expiratory flushing of airways (EFLAW).

\begin{tabular}{lccccc}
\hline & \multicolumn{5}{c}{ EFLAW EFLAW EFLAW EFLAW } \\
& NMV & $2 \mathrm{~Hz}$ & $4 \mathrm{~Hz}$ & $8 \mathrm{~Hz}$ & c.f. \\
\hline $\mathrm{PaCO}_{2}(\mathrm{mmHg})$ & 34 & 33 & 33 & 34 & 34 \\
$\mathrm{~V}_{\mathrm{r}}(\mathrm{ml})$ & 196 & 142 & 151 & 152 & 157 \\
$\mathrm{P}_{\text {peak }}\left(\mathrm{cmH}_{2} \mathrm{O}\right)$ & 9.2 & 7.2 & 7.4 & 7.4 & 7.8 \\
$\mathrm{P}_{\text {mean }}\left(\mathrm{cmH}_{2} \mathrm{O}\right)$ & 2.4 & 1.8 & 1.9 & 2.0 & 2.2 \\
\hline
\end{tabular}

c.f. $=$ continuous flow, $\mathrm{n}=6$.

of expiration $(4 \mathrm{~Hz})$ the flow recorded from the Servo Ventilator showed the pattern of Fig. 3. The volume of gas used for flushing was about 1.5 times as large as the airway dead space measured with the single breath test during normal mechanical ventilation (NMV). Fig. 4 shows the SBT-CO ${ }_{2}$ recorded during EFLA. This type of recording allows a detailed analysis of $\mathrm{CO}_{2}$ elimination.

Table 2 shows that the tidal volume could be reduced to about $3 / 4$ of that during NMV, and peak pressure almost as much. Whether flushing was performed as pulses of 2 to $8 \mathrm{~Hz}$ or as a continuous flow (c.f.) was of no significance. Fig. 4 shows that most of the $\mathrm{CO}_{2}$ flushed out during EFLA was eliminated during the first pulses. If the flushing volume was increased to 2.5 times the airway dead space, this did not contribute significantly to the efficiency of EFLA.

\section{Measurement of airway pressure during $\mathrm{HFJV}$}

Healthy dogs and dogs with severe respiratory distress syndrome (RDS) after lung lavage (8) were ventilated with HFJV (9). It was found that pressure measured with a Millar catheter in the airway differed dramatically from that of the retrograde catheter until the Millar tip transducer was advanced down to the level

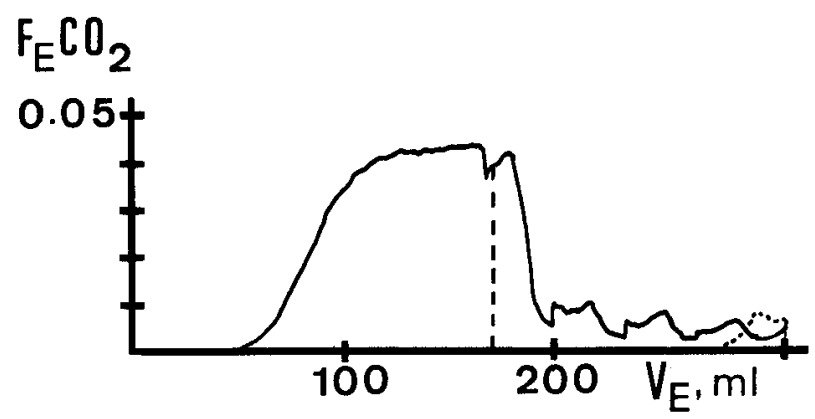

Fig. 4. The SBT- $\mathrm{CO}_{2}$ displays fraction of expired $\mathrm{CO}_{2}$ over expired volume. The initial part corresponding to the normal expiration resembles a normal SBT- $\mathrm{CO}_{2}$ (to the left of the broken line). Thereafter, additional $\mathrm{CO}_{2}$ is expired during the "mini-expirations" caused by EFLA pulses. The fraction of $\mathrm{CO}_{2}$ falls to almost zero after some pulses, indicating that EFLA efficiently eliminates $\mathrm{CO}_{2}$ from the airway. 
of lobar or segmental bronchi. At this level no numerical or visual difference between the two pressures was observed. The Millar catheter placed at this level was therefore used to determine the bronchial pressure $\left(\mathrm{P}_{\mathrm{br}}\right)$ to which peripheral airways are exposed during HFJV. The pressure measured in the tracheal tube $\left(\mathrm{P}_{\mathrm{tr}}\right)$ showed, during the phase of jet insufflation, a drop rather than a peak (Fig, 5). The pressure measured as lateral pressure in the tracheal tube underestimates the peak and mean airway pressure to which the peripheral airways are exposed. The error is due to the Bernoulli effect. When high flow rates are used, which is necessary in HFJV in RDS the underestimation becomes severe.

\section{DISGUSSION}

The methods discussed refer to HFJV or combined HFJV performed with a high frequency unit (HFU) connected to the Servo Ventilator 900 C. A prototype of the HFU that will be commercially available was used. This prototype permits a greater variety of settings but functions in principle as the commercial unit. The $\mathrm{CO}_{2}$ analyzer is a standard option of the ventilator system which may be used either in the conventional way when ventilation is based on an ordinary mode supplemented by a high frequency component during the expiratory phase (Fig. 2) - or in a non-conventional way when ventilation is based on high frequency jet ventilation (Fig. 1) with or without supplementary inflations of low frequency (10).

A system based upon the Servo Ventilator with the

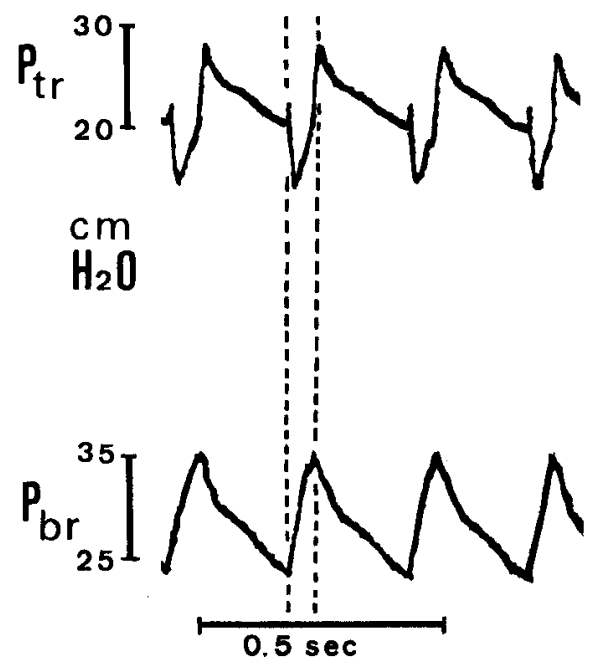

Fig. 5. When pressure is recorded in the bronchi with a Millar catheter and in the trachea (as in Fig. 1), a large difference is observed during the period of the jet pulse. Tracheal pressure falls due to the Bernoulli effect, whereas bronchial pressure increases.
HFU makes measurement and monitoring possible according to the examples presented.

During HFJV the ventilator may be set so that a certain minute ventilation is produced. The ventilation is monitored and guarded by the alarm system of the Servo Ventilator. At moderately high frequencies, such as $2 \mathrm{~Hz}$, the deadspace fraction of the minute ventilation is of the same magnitude as during normal mechanical ventilation (NMV) when a system such as the present one is used. Variations in minute ventilation at a constant frequency in this range have a physiological significance as during NMV, and will result in a change of $\mathrm{CO}_{2}$ elimination and $\mathrm{PaCO}_{2}$.

At higher frequencies the deadspace fraction of gas supplied as a jet increases greatly. Should it, for clinical reasons, be desired to enhance $\mathrm{CO}_{2}$ production, a small reduction in frequency is then often more rational than the large increase in minute ventilation that would be needed. At high frequencies modification of minute ventilation will result in variation of the auto-PEEP effect which is a typical phenomenon of HFV (11-13). The auto-PEEP effect is due to the fact that expiration time becomes too short for a full expiration when the frequency is high. Above a certain frequency a change in ventilation will have a strong influence on auto-PEEP.

The rationale for the use of HFJV is to provide optimal alveolar ventilation and to maintain alveolar pressure at a level around which only small fluctuations occur. This calls for the use of rather high frequencies. At these frequencies the physiological effects of variations in minute ventilation, frequency and external PEEP are difficult to predict. An increase in total ventilation may, for example, have a small effect upon alveolar ventilation, but may reduce alveolar perfusion due to the associated auto-PEEP effect. A minor change in patient status, such that physiological deadspace fraction changes $5 \%$ (say from 75 to $80 \%$ ), will cause a major change in gas exchange. We may conclude that safety margins are small during HFV, and that the effects of variations in patient status and in ventilator setting may be large and unpredictable. Monitoring, essential in NMV, is vital in HFJV.

Continuous monitoring of $\mathrm{CO}_{2}$ elimination in $\mathrm{HFJV}$ is possible in either of the two modes described in Fig. 1 and 2. When HFJV was instituted, we found that it was easy to find a setting that maintained isocapnia under guidance of $\mathrm{CO}_{2}$ elimination. The same is true of resetting the HFJV mode. When steady state has been established, any sudden disturbance of alveolar ventilation and/or perfusion will display as a variation in $\mathrm{CO}_{2}$ elimination. (Slow variations in physiology may be detected by measurements of blood gases or related methods). 
An alternative to measurements of $\mathrm{CO}_{2}$ elimination is to monitor $\mathrm{O}_{2}$ uptake. This involves more complicated instrumentation and is notoriously difficult when extraction of $\mathrm{O}_{2}$ is low and the fraction of $\mathrm{O}_{2}$ is high in inspired gas, which is normally the case in HFJV. In conclusion, monitoring of $\mathrm{CO}_{2}$ elimination is essential in HFJV and feasible with standard equipment.

Studies of gas exchange in HFJV may be performed according to the methods described. It should be noted that the effects of HFJV depend to a large extent on factors such as the geometry of the jet system, the shape of the pulses and their I:E ratio. The performance of each HFJV system should thus be analyzed separately.

Airway pressure measurement constitutes an important problem in HFV. A jet is directed downstream in the airway, and one must measure the pressure below the jet opening. A lumen for pressure monitoring ending close to the tip of the tracheal tube has obvious advantages. However, the Bernoulli effect is large when high flow rates are applied. This wellknown effect is often neglected in studies of HFJV. The use of a pressure catheter ending several centimeters below the tracheal tube is recommended for accurate determination of the pressure to which airways and peripheral lung are subjected in HFJV. The use of such catheters is, however, not practical in clinical routine. Tracheal tube tip measurements may be used if the magnitude of the Bernoulli effect under the prevailing circumstances is understood.

The auto-PEEP effect may be harmful or may be one of the therapeutic goals of HFJV. It needs to be quantified. The best way to achieve this is to halt the HFU and simultaneously close the valves of the Servo Ventilator. This causes a no-flow period; the prevalent auto-PEEP is then reflected in the recoil pressure of the respiratory system and can be determined from the airway pressure. This is possible by pressing the end-expiratory pause button of the Servo Ventilator with the HFV unit.

Another aspect of lung distension during HFJV is assessed by measuring FRC. If the lung volume is equilibrated with a certain concentration of a tracer gas such as $\mathrm{SF}_{6}$, and washed out from a given moment, the volume of $\mathrm{SF}_{6}$ eliminated from that moment allows determination of FRC $(2,3)$. The principle for measurement of gas elimination illustrated in Fig. 1 has been proven to work for such studies. We have no data to demonstrate the accuracy in HFJV of FRG measurements based upon the principle.

\section{ACKNOWLEDGEMENTS}

This study was supported by The Swedish National Association against Chest and Heart Diseases and by the Swedish Medical Research Council (grant 02872).

\section{REFERENCES}

1. Olsson S-G, Fletcher R, Jonson B, Nordström L, Prakash O. Clinical studies of gas exchange during ventilatory support - a method using the Siemens-Elema $\mathrm{CO}_{2}$-analyzer. $\mathrm{Br} \mathcal{F}$ Anaesth 1980: 52: 491-499.

2. Jonmarker C, Jansson L, Jonson B, Larsson A, Werner O. Measurement of functional residual capacity by sulfur hexafluoride washout. Anesthesiology 1985: 63: 89-95.

3. Larsson A, Linnarsson D, Jonmarker C, Jonson B, Larsson H, Werner $O$. Measurement of lung volume by sulfur hexafluoride washout during spontaneous and controlled ventilation; further development of a method. Anesthesiology 1987: 67: 543-550.

4. Fletcher R, Jonson B, Cumming G, Brew J. The concept of dead space with special reference to the single breath test for $\mathrm{CO}_{2} . \mathrm{Br}$ J Anaesth 1981: 53: 77.

5. Macklem P T, Mead J. Resistance of central and peripheral airways measured by a retrograde catheter. 7 Appl Physiol 1967 : 22: 395-401.

6. Malmkvist G, Fletcher R, Drefeldt B, Jonson B, Lührs C, Mori N. Deadspace during high frequency jet ventilation: the effects of frequency, inspiratory time and entrainment. Clin Phys 1985: 5, suppl. 3: 143-146.

7. Jonson B et al. Purge expiratoire des voies aériennes. In: Fonction diaphragmatique. Travail respiratoire. Paris: Expansion Scientifique Francaise, 1987: 195-201.

8. Lachmann B, Danzmann E, Haendly B, Jonson B. Ventilator settings and gas exchange in respiratory distress syndrome. In: Prakash $\mathrm{O}$, ed. Applied physiology in clinical respiratory care. The Hague: Martinus Nijhoff, 1982: 141-176.

9. Jonson B, Evander E, Lachmann B, Wollmer P. Pathophysiological considerations on special modes of ventilation in severe respiratory distress syndrome. In: Gasparetto A, ed. Int. Resuscitation Days. Elsevier Science Publishers B.V. (Biomedical Division), 1986.

10. Jonson B, Lachmann B. High frequency jet ventilation in dogs with respiratory distress syndrome. In preparation.

11. Carlon G C, Ray G, Klain M, McCormack P M. Highfrequency positive-pressure ventilation in management of a patient with bronchopleural fistula. Anesthesiology 1980: 52: $160-162$.

12. Rouby J J, Fusciardi J, Bourgain J L, Viars P. High-frequency jet ventilation in postoperative respiratory failure: determinants of oxygenation. Anesthesiology 1983: 59: 281-287.

13. Mal H, Rouby J J, Benhamou D, Viars P. High frequency jet ventilation in acute respiratory failure: which ventilator settings? Br $\mathcal{J}$ Anaesth 1986: 58: 18-23.

\section{Address:}

Prof. Dr.Björn Fonson

Department of Clinical Physiology

University Hospital

S-221 85 Lund, Sweden 